\title{
Mitochondrial activity detected by cantilever based sensor
}

\author{
Petar Stupar $^{1}$, Wojciech Chomicki ${ }^{1}$, Caroline Maillard ${ }^{1}$, David Mikeladze ${ }^{2}$, Aleksandar Kalauzi ${ }^{3}$, \\ Ksenija Radotić ${ }^{3}$, Giovanni Dietler ${ }^{1}$, and Sandor Kasas ${ }^{1,4}$ \\ ${ }^{1}$ Laboratory of Physics of Living Matter, EPFL, Lausanne, Switzerland \\ ${ }^{2}$ School of Natural Sciences and Engineering, Ilia State University, Tbilisi, Georgia \\ ${ }^{3}$ Institute for multidisciplinary research, University of Belgrade, Belgrade, Serbia \\ ${ }^{4}$ Plateforme de Morphologie, Université de Lausanne, Switzerland \\ Correspondence to: Sandor Kasas (sandor.kasas@epfl.ch)
}

Received: 31 October 2016 - Revised: 26 January 2017 - Accepted: 28 February 2017 - Published: 14 March 2017

\begin{abstract}
Our team recently demonstrated that cantilever based devices can detect signature of life in a chemistry independent manner. In this technique, the organism of interest is attached onto a classical AFM cantilever. If alive, it induces nanometre scale oscillations of the cantilever that disappear once the organism is killed. The technique was successfully used on bacteria, yeast, vegetal and mammalian cells. In this work we demonstrate that the method can also be applied to sub-cellular organelles, such as mitochondria. Mitochondria are involved in cellular energy production and are present in most eukaryotic cells. Nowadays, it is believed that mitochondria were originally prokaryotes that colonized eukaryotic cells and that live in an endosymbiotic way ever since. Here we present that mitochondria are also animated by nanometre scale oscillations that depend on their metabolic state and that stop once they are inhibited. This observation opens novel avenues to investigate the numerous mitochondria-related diseases in humans.
\end{abstract}

\section{Introduction}

Mitochondria are $0.7-3 \mu \mathrm{m}$ sized sub-cellular organelles that contain their own DNA, and it is nowadays believed that they were independent prokaryotic cells that long time ago colonized eukaryotes (Yang et al., 1985). Mitochondria live with eukaryotes in an endosymbiotic manner and are involved in numerous key physiological processes, such as energy production and regulation, signalling and programmed cellular death. Altered mitochondrial function is a key underlying mechanism of many pathological states, such as cardiac diseases, diabetes and numerous other neurological conditions (Schapira, 2012). Precise assessment of mitochondrial function is necessary to understand those underlying mechanisms and the aging process. Furthermore, growing literature indicates that mitochondria are also targeted by environmental pollutants, making them an important subject in environmental toxicology and health research (Meyer et al., 2013). However, the diagnosis of mitochondrial diseases is a complicated, expensive, and time consuming process that in- volves mitochondrial isolation, DNA extraction and sequencing. Among the tools for probing mitochondrial properties, measurements of oxygen consumption and ATP production have been used to assess the function of freshly isolated mitochondria (Lanza and Nair, 2009).

Few years ago, our team noticed that atomic force microscopy (AFM) cantilevers can be used as nanomotion sensors to detect subtle oscillations that characterize living organisms. Despite the fact that the origin of these oscillations is still not fully understood, we could observe that the amplitude (variance) of these oscillations reflect the metabolic state of the organism of interest. We also noticed that these nanoscale oscillations exist in every living biological sample we tested so far (bacteria, yeast, vegetal and mammalian cells) (Longo et al., 2013; Aghayee et al., 2013; AlonsoSarduy et al., 2014; Kasas et al., 2015). In an attempt to simplify mitochondrial disease diagnostic, we carried out preliminary experiments to explore the putative nanoscale oscillation pattern of native mitochondria. The experiments consisted in attaching isolated mitochondria onto an AFM can- 

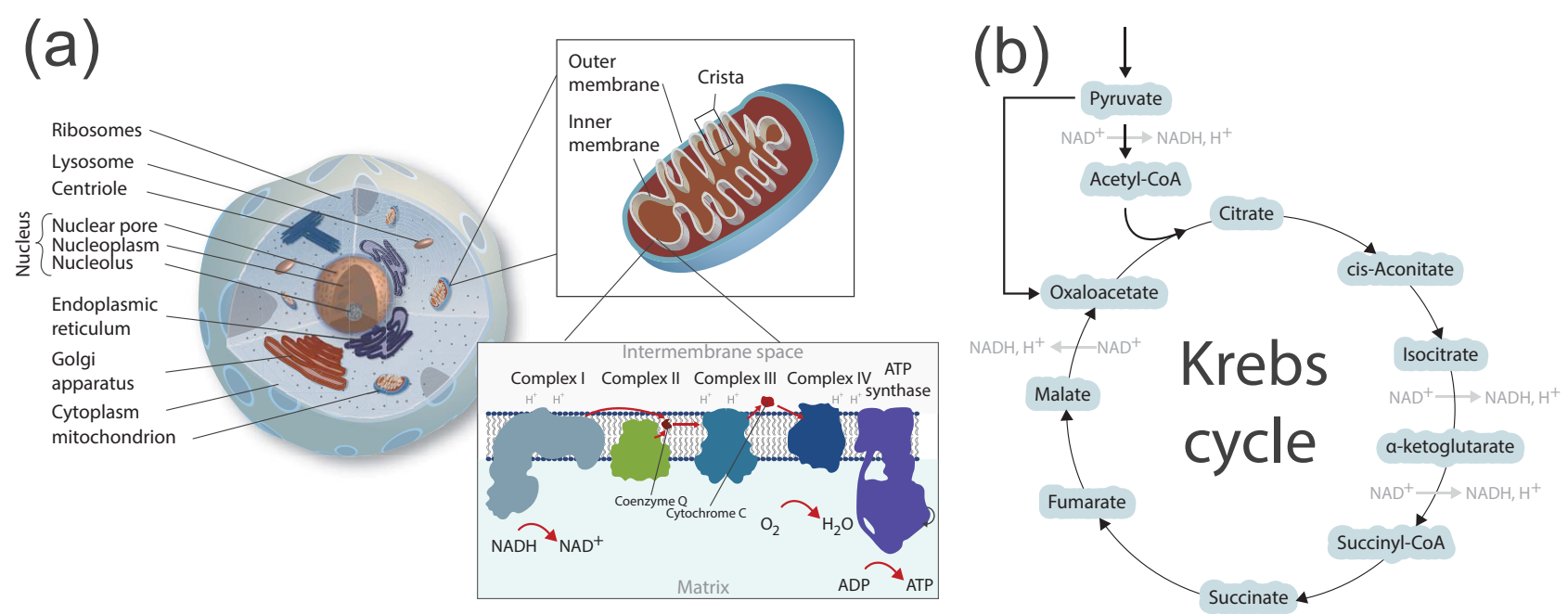

Figure 1. Panel (a): Schematic of a mammalian cell with labelled organelles, zoom on the mitochondrion showing its structure and most important components of the inner membrane (Complexes I-IV and ATP synthase). Panel (b): Krebs cycle (citric acid cycle) showing the sequence of metabolic reactions that generate energy within mitochondria.

tilever and recording its oscillations as a function of exposure to different chemicals.

Enzymes within the mitochondrial matrix (Fig. 1a) are designed to oxidize the added substrates, in a cyclic manner (Fig. 1b), so that every product of a reaction is a potential substrate for another reaction. In our experiments, human embryonic kidney cell mitochondria were submitted to malate, pyruvate, ADP, sodium azide and rotenone. Malate and pyruvate are the substrates involved in the citric acid cycle (Kerbs' cycle), and their oxidation provides energy conserved in the structure of molecules like NADH and $\mathrm{FADH}_{2}$. These energy carriers lose energy by interacting with complexes embedded in the mitochondrial inner membrane. Complexes (I-IV) transfer energy by passing along the electrons in a process called the electron transport system. Starting with re-oxidation of a NADH molecule, a pair of electrons is passed through a series of carriers to coenzyme $\mathrm{Q}$, forcing protons from the matrix into the intermembrane space. The electrons are then passed through Complex III, forcing more protons out, and finally to Complex IV, where oxygen is reduced to water by using the electrons. In this process, some energy is released as heat, while the majority is conserved within the created proton gradient. The last link in the chain of complexes is the ATP synthase (F0 and F1 subunits). It holds a proton channel, where protons are pumped through, allowing energy for the synthesis of ATP by rotating F1 subunit and changing the conformation of binding sites where ADP molecules react with available phosphate.

Apart from the substrates, our experiments consisted in adding inhibitors, sodium aizde and rotenone. Sodium azide inhibits the ATPase activity of F1 subunit, but has no inhibitory effect on ATP synthesis (Hong and Pedersen, 2008). Rotenone is another inhibitor and it acts by inhibiting NADH dehydrogenase or Complex I of the electron transport chain (Li et al., 2003).

The oscillation patterns that we observed with mitochondria on the cantilever were highly dependent on the chemicals to which they were exposed. These preliminary experiments indicate that mitochondria, similarly to bacteria, yeast, vegetal and mammalian cells also promote oscillations of the cantilever to which they are attached.

\section{Materials and Methods}

\subsection{Solutions and buffers}

Buffer 1 (HB): $210 \mathrm{mM}$ mannitol, $70 \mathrm{mM}$ sucrose, $5 \mathrm{mM}$ HEPES (pH 7.12). Buffer 2 (HBS): HB +1 mM EGTA $+1 \times$ Roche's Complete Mini EDTA-free protease inhibitor cocktail (1 tablet dissolved in $10 \mathrm{~mL}$ of buffer). $50 \%$ Percoll: Percoll (Sigma P1644) diluted 1 to 1 with HB. Percoll solutions (22 and 15\%): The 50\% Percoll stock is used to prepare all other Percoll solutions; to that effect dilutions of the $50 \%$ Percoll solutions are done in HBS.

Substrate solutions were prepared in advance and stored at $-20^{\circ} \mathrm{C}$. All substrates were dissolved in the working buffer $(125 \mathrm{mM} \mathrm{KCl}, 10 \mathrm{mM}$ TRIS, $0.1 \mathrm{mM}$ EGTA, $1 \mathrm{mM}$ $\mathrm{KH} 2 \mathrm{PO} 4, \mathrm{pH}$ 7.4), while rotenone was initially dissolved in $50 \%$ ethanol and then diluted with the buffer. Malate and pyruvate were used in $5 \mathrm{mM}$ final concentration, while ADP was added in $1 \mathrm{mM}$ final concentration. Sodium azide was used in $10 \mathrm{mM}$, while rotenone in $1 \mu \mathrm{M}$.

\subsection{Mitochondria isolation}

Mitochondria were isolated according to a modified protocol (Kristián et al., 2006). Human embryonic kidney cells 

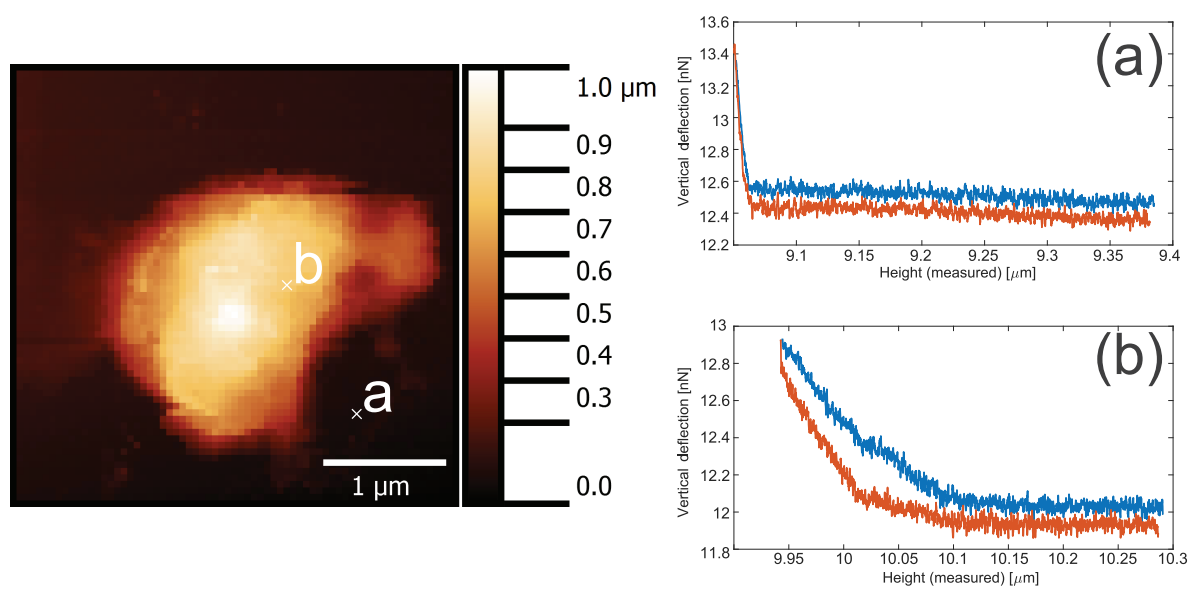

Figure 2. The AFM image of isolated mitochondrion attached to glass, in buffered conditions. Structure shows $2.5 \mu \mathrm{m}$ length, $2 \mu \mathrm{m}$ width and $1.1 \mu \mathrm{m}$ height. Force-distance curves from positions marked on the AFM image are shown on the right. The top figure (a) shows the stiffness of the substrate, while the other one (b) shows the softness of the biological structure.

(293T) were grown to approximately $85 \%$ in DMEM. Plates were placed on ice and washed twice with $8 \mathrm{~mL}$ of ice cold HB. Cells were collected by scraping directly in the Dounce Tissue Grinder and homogenized well. The obtained homogenate was spun at $1500 \mathrm{~g}$ for $3 \mathrm{~min}$. The supernatant was spun at $13000 \mathrm{~g}$ for $17 \mathrm{~min}$ and the pellet was re-suspended in $1.4 \mathrm{~mL}$ of HBS. $5 \mathrm{~mL}$ centrifuge tubes with a discontinued Percoll gradient were used $(1 \mathrm{~mL}$ of $50 \%$ Percoll solution followed by layering $3 \mathrm{~mL}$ of $22 \%$ Percoll solution). To the re-suspended pellet, $0.6 \mathrm{~mL}$ of $50 \%$ Percoll (final concentration of Percoll $15 \%$ ) was added and layered $1 \mathrm{~mL}$ of the $15 \%$ Percoll sample on top of the described 50-22\% gradient. This was spun at $30700 \mathrm{~g}$ for $6 \mathrm{~min}$ and mitochondria were recovered from 50-22\% Percoll interface. Mitochondria were finally washed by making 1 to 10 dilution with HBS and centrifuging at $15600 \mathrm{~g}$ for $30 \mathrm{~min}$.

\subsection{AFM imaging}

Atomic Force Microscopy measurements were made using Nanowizard III AFM from JPK Instruments (Berlin, Germany), coupled with a Zeiss Axiovert inverted optical microscope. A fluid cell was incorporated and all the images were obtained in buffered conditions. Images were collected using DNP-10 (Bruker) cantilevers, with a nominal spring constant of $0.06 \mathrm{~N} \mathrm{~m}^{-1}$. Quantitative imaging mode has been used for the image acquisition, Gwyddion (v. 2.36) software for flattening and no further image processing has been carried out.

\subsection{Mitochondria attachment onto AFM cantilevers}

Mitochondria were attached to cantilevers using glutaraldehyde functionalization and the following procedure. Conventional NP-O10 cantilevers (Bruker) were functionalized using $0.5 \%$ glutaraldehyde for $10 \mathrm{~min}$. All cantilevers were washed afterwards, and placed to dry for the following $4 \mathrm{~min}$.
A droplet of solution containing concentrated mitochondria was placed onto the functionalized cantilevers and left for $30 \mathrm{~min}$. After the attachment, the cantilevers were mounted and positioned inside a fluid cell, together with the working buffer.

\subsection{Data acquisition and processing}

Real time deflection of the cantilever was collected at the sampling frequency of $20 \mathrm{kHz}$. The signal was first flattened using a running window of $200 \mathrm{~s}$. A linear fit was applied to each window and the raw signal was subtracted, so the outcome is a de-trended signal, centred around zero. Such signal was further processed by applying the moving average of $4 \mathrm{~s}$. Finally, variance was calculated in the similar manner, using a running window of $10 \mathrm{~s}$. Variance signal was used for line and bar plots in the presented figures. Error bars in Fig. 5 represent the standard error of the mean.

\section{Results}

Before exploring the mitochondrial sample with our technique, we imaged the attached mitochondria on a glass substrate in liquid media. Figure 2 shows the obtained image with examples of force-distance curves at two different positions, one on the mitochondrion and the other one on the support. Curves show a typical response of the cantilever in contact with a soft and a hard sample, respectively.

Next, freshly isolated mitochondria were attached to the cantilever sensor and introduced into the analysis chamber. Real-time cantilever deflection recordings were made while mitochondria were submitted to different conditions by using the fluid cell that allowed us the fluid exchange. The first media to make a measurement was a plain buffer, after which we introduced substrates: malate and pyruvate. With 


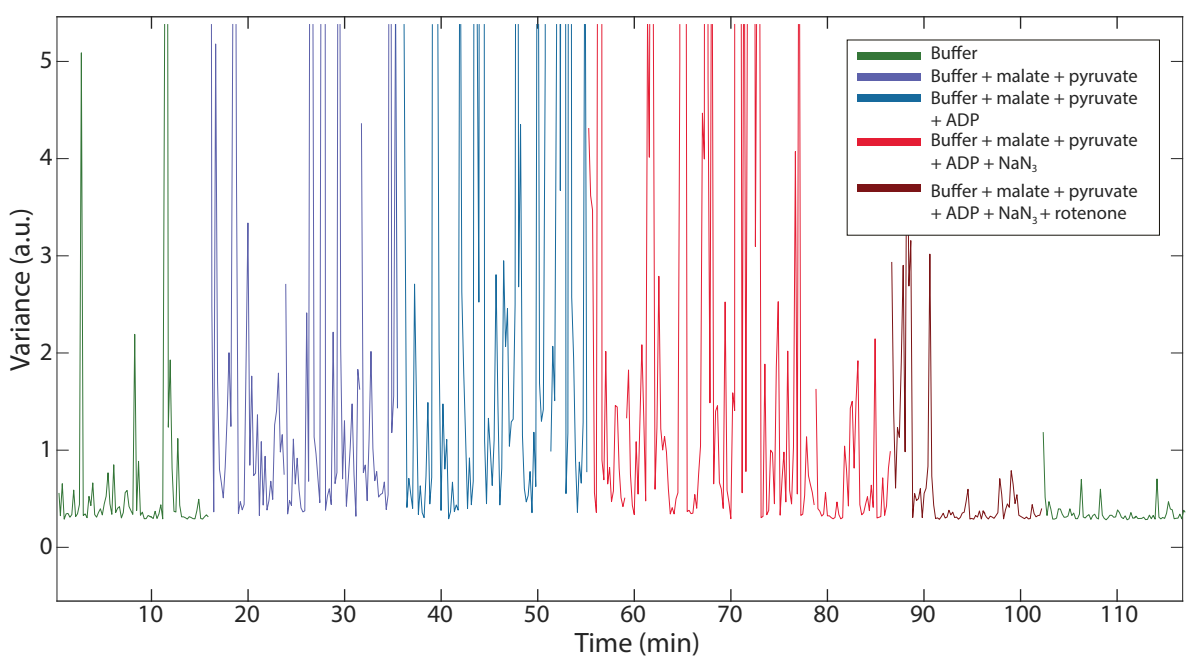

Figure 3. The evolution of variance throughout the experiment. Variance was calculated from the deflection data for each measurement. Different colours represent different conditions to which mitochondria were subjected. In each condition a new substance was added (substrates: malate, pyruvate, ADP; inhibitors: $\mathrm{NaN}_{3}$, rotenone). Variance increases with the addition of substrates and decreases in presence of the inhibitors.
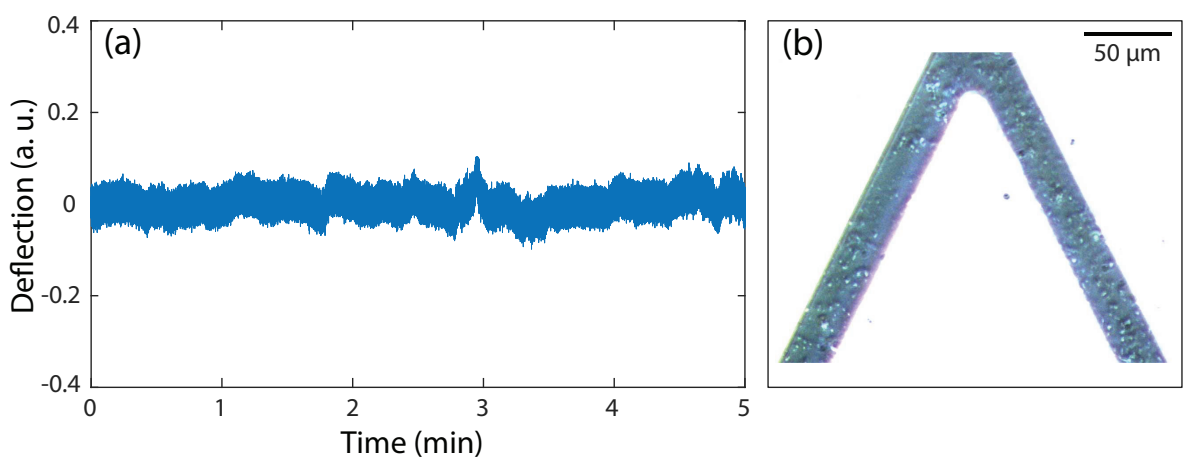

Figure 4. Panel (a): Deflection of the cantilever with mitochondria attached. The recordings were made while the buffer with substrates (malate, pyruvate, ADP) was present in the analysis chamber. Figure shows a section of 5 min oscillations. Panel (b): A typical image of mitochondria attached to the cantilever.

introduction of the substrates, the oscillations increased, as presented on the variance plot in Fig. 3. Oxygen consumption in this condition reflects state 2 respiration of mitochondria, specific to Complex I. Then, we introduced ADP to the medium to stimulate further the respiration in the presence of malate and pyruvate. In this condition, state 3 respiration, specific to Complexes I and II, appears to keep oscillations of the sensor increased. The example of oscillations, plotted as a real-time deflection, is presented on Fig. 4. We continued the experiment by adding sodium azide and measuring the response. The oscillations showed a slow decrease, and were completely reduced only after the injection of media containing rotenone.

Lastly, Fig. 5 shows the variance averaged over $15 \mathrm{~min}$ intervals within different conditions. Two sets of bars are presented, one with the control cantilever (functionalized, but with no mitochondria attached), and the other one with mi- tochondria attached. Each set is normalized to the first measurement (buffer) separately, and represents the percentage change of variance once the substrates (and the inhibitor) are added. While the control cantilever oscillations remained within the error interval, the one with mitochondria attached showed more than two times increase in oscillations in the presence of metabolic substrates compared to the buffer only. Values suggest that mitochondria do couple with the cantilever and induce oscillations greatly above the baseline thermal motion, when activated by substrates. These oscillations dropped with the introduction of the inhibitor, suggesting the complete inhibition of mitochondrial activity.

\section{Discussion}

In this article we showed how nanometre scale oscillations arising from mitochondrial processes coupled with the can- 

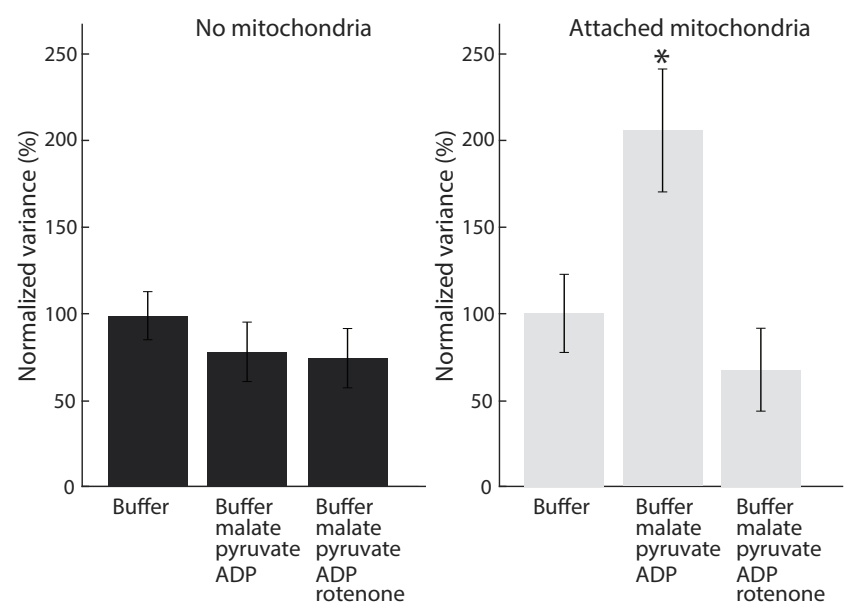

Figure 5. Normalized and averaged variance over the $15 \mathrm{~min}$ intervals in conditions of a plain buffer, buffer with substrates and lastly, with the inhibitor. Dark grey represents values without any mitochondria on the cantilever as a control, while light grey values show variance changes with the attached mitochondria. The twosample $t$ test rejected the null hypothesis at the $5 \%$ significance level $(p \leq 0.05)$ only in the case of attached mitochondria with the addition of substrates.

tilever and produced motion. Conventional FFT analysis, unfortunately, could not produce any distinct frequency components specific to the oscillations in different metabolic conditions. Therefore, we believe variance evolution is the best parameter to describe dynamic changes in oscillations. As showed on Fig. 3, switching from plain buffer into a buffer with metabolic substrates caused oscillations to increase. Those oscillations were amplified with the addition of a diphosphate necessary for the series of conformational changes that precedes the ATP synthesis. Sodium azide is an inhibitor that acts by confining the activity of ATPase, without stopping the ATP synthesis coupled mechanically to the proton motive force across the inner membrane by the rotation of the subdomain ensemble (Bowler et al., 2006). Furthermore, some reports show that the presence of inorganic phosphate could tamper the ability of azide as an inhibitor (Minkov and Strotmann, 1989). Therefore, the observed slow and incomplete decrease of variance could be attributed to azide not being as potent inhibitor in our experimental setup. Rotenone, a specific NADH dehydrogenase inhibitor, stops the electron transfer to coenzyme Q. This creates reactive oxygen species which can damage DNA and other components of mitochondria. This could suggest why in the presence of rotenone in our experiments, oscillations of the cantilever dropped to lowest values.

Considering our previous work, it is not surprising that a cantilever with the attached mitochondria oscillates more than a control in the presence of metabolic substrates. The observed increase could be attributed to the complex dynamics of mitochondrial metabolic system.

\section{Conclusions}

Mitochondrial diseases are a public health issue nowadays and their diagnostic is unfortunately still a complicated, long, and expensive process. In these experiments we demonstrated that the activity of native mitochondria can be detected, like in the case of other prokaryotes. We have also shown that their oscillation pattern is affected by the chemicals to which they were exposed. Having in mind these two facts, it can be speculated that the oscillation pattern of "healthy" and "diseased" mitochondria could also differ. Likewise, it could be expected that they react differently upon appropriate chemical exposure. Such a possibility would open novel avenues in mitochondrial disease diagnosis and research. Even if for the moment we did not explore these interesting hypotheses, the present work suggests that continuing the investigation might lead to a great benefit in terms of diagnostic speed and cost. The next step is to extend our studies to mitochondria isolated from patients suffering from mitochondrial diseases.

Data availability. All datasets used in the manuscript can be claimed on request to the corresponding author.

Competing interests. The authors declare that they have no conflict of interest.

Acknowledgements. Petar Stupar, Giovanni Dietler, and Sandor Kasas were funded by the Swiss National Grant 200021-144321 and also acknowledge the support of Gebert Rüf Stiftung GRS024/14 and Centre Cooperation \& Developpement Program Seed Money 2016. Aleksandar Kalauzi and Ksenija Radotić would like to acknowledge grants ON173017 and III45012 from the Ministry of education, science and technological development of the Republic of Serbia.

Edited by: M. K. Ghatkesar

Reviewed by: two anonymous referees

\section{References}

Aghayee, S., Benadiba, C., Notz, J., Kasas, S., Dietler, G. and Longo, G.: Combination of fluorescence microscopy and nanomotion detection to characterize bacteria, J. Mol. Recognit., 26, 590-595, doi:10.1002/jmr.2306, 2013.

Alonso-Sarduy, L., De Los Rios, P., Benedetti, F., Vobornik, D., Dietler, G., Kasas, S., and Longo, G.: Real-time monitoring of protein conformational changes using a nano-mechanical sensor, PloS One, 9, e103674, doi:10.1371/journal.pone.0103674, 2014.

Bowler, M. W., Montgomery, M. G., Leslie, A. G. W., and Walker, J. E.: How azide inhibits ATP hydrolysis by the F-ATPases, P. Natl. Acad. Sci. USA, 103, 8646-8649, doi:10.1073/pnas.0602915103, 2006. 
Hong, S. and Pedersen, P. L.: ATP Synthase and the Actions of Inhibitors Utilized To Study Its Roles in Human Health, Disease, and Other Scientific Areas, Microbiol. Mol. Biol. Rev., 72, 590641, doi:10.1128/MMBR.00016-08, 2008.

Kasas, S., Ruggeri, F. S., Benadiba, C., Maillard, C., Stupar, P., Tournu, H., Dietler, G., and Longo, G.: Detecting nanoscale vibrations as signature of life, P. Natl. Acad. Sci. USA, 112, 378381, doi:10.1073/pnas.1415348112, 2015.

Kristián, T., Hopkins, I. B., McKenna, M. C., and Fiskum, G.: Isolation of mitochondria with high respiratory control from primary cultures of neurons and astrocytes using nitrogen cavitation, J. Neurosci. Methods, 152, 136-143, doi:10.1016/j.jneumeth.2005.08.018, 2006.

Lanza, I. R. and Nair, K. S.: Functional Assessment of Isolated Mitochondria In Vitro, Methods Enzymol., 457, 349-372, doi:10.1016/S0076-6879(09)05020-4, 2009.

Li, N., Ragheb, K., Lawler, G., Sturgis, J., Rajwa, B., Melendez, J. A., and Robinson, J. P.: Mitochondrial Complex I Inhibitor Rotenone Induces Apoptosis through Enhancing Mitochondrial Reactive Oxygen Species Production, J. Biol. Chem., 278, 85168525, doi:10.1074/jbc.M210432200, 2003.
Longo, G., Alonso-Sarduy, L., Rio, L. M., Bizzini, A., Trampuz, A., Notz, J., Dietler, G., and Kasas, S.: Rapid detection of bacterial resistance to antibiotics using AFM cantilevers as nanomechanical sensors, Nat. Nanotechnol., 8, 522-526, doi:10.1038/nnano.2013.120, 2013.

Meyer, J. N., Leung, M. C. K., Rooney, J. P., Sendoel, A., Hengartner, M. O., Kisby, G. E., and Bess, A. S.: Mitochondria as a Target of Environmental Toxicants, Toxicol. Sci., 134, 1-17, doi:10.1093/toxsci/kft102, 2013.

Minkov, I. B. and Strotmann, H.: The effect of azide on regulation of the chloroplast H+-ATPase by ADP and phosphate, Biochim. Biophys. Acta BBA - Bioenerg., 973, 7-12, doi:10.1016/S00052728(89)80394-9, 1989.

Schapira, A. H.: Mitochondrial diseases, The Lancet, 379, 1825 1834, doi:10.1016/S0140-6736(11)61305-6, 2012.

Yang, D., Oyaizu, Y., Oyaizu, H., Olsen, G. J., and Woese, C. R.: Mitochondrial origins, P. Natl. Acad. Sci. USA, 82, 4443-4447, 1985. 